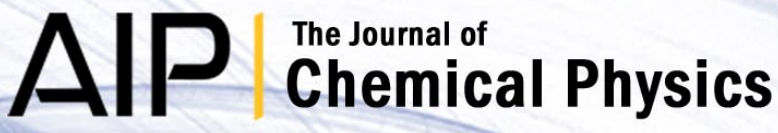

Time-dependent quantum transport: An efficient method based on Liouville-von-Neumann equation for single-electron density matrix

Hang Xie, Feng Jiang, Heng Tian, Xiao Zheng, Yanho Kwok et al.

Citation: J. Chem. Phys. 137, 044113 (2012); doi: 10.1063/1.4737864

View online: http://dx.doi.org/10.1063/1.4737864

View Table of Contents: http://jcp.aip.org/resource/1/JCPSA6/v137/i4

Published by the AIP Publishing LLC.

Additional information on J. Chem. Phys.

Journal Homepage: http://jcp.aip.org/

Journal Information: http://jcp.aip.org/about/about_the_journal

Top downloads: http://jcp.aip.org/features/most_downloaded

Information for Authors: http://jcp.aip.org/authors

\section{ADVERTISEMENT}

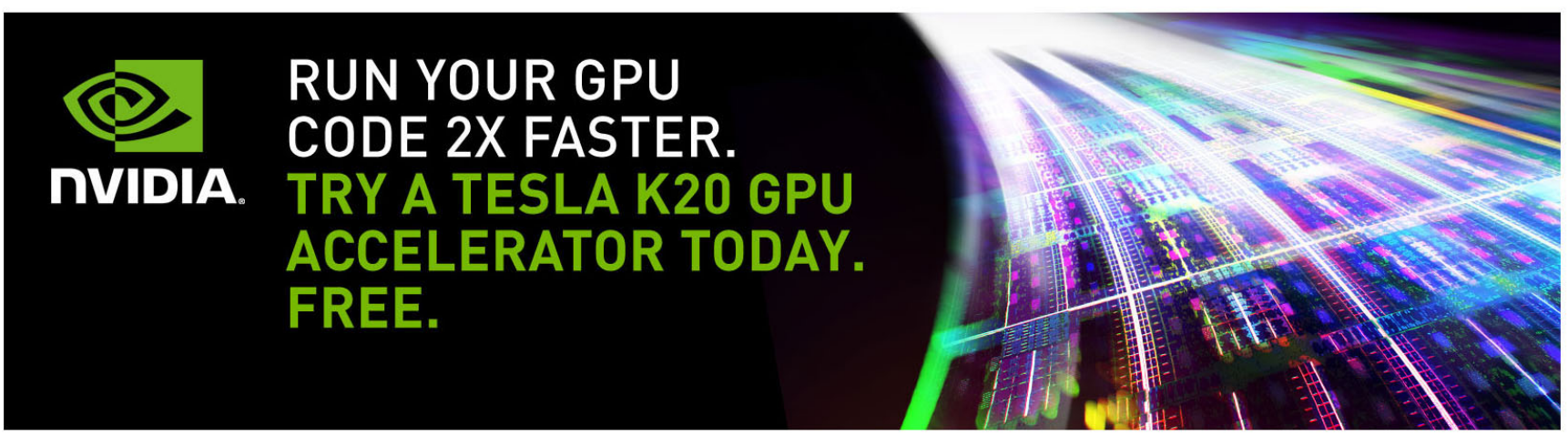




\title{
Time-dependent quantum transport: An efficient method based on Liouville-von-Neumann equation for single-electron density matrix
}

\author{
Hang Xie, ${ }^{1}$ Feng Jiang, ${ }^{2}$ Heng Tian, ${ }^{1}$ Xiao Zheng, ${ }^{3}$ Yanho Kwok, ${ }^{1}$ Shuguang Chen, ${ }^{1}$ \\ ChiYung Yam, ${ }^{1}$ YiJing Yan, ${ }^{2,3}$ and Guanhua Chen ${ }^{1, a)}$ \\ ${ }^{1}$ Department of Chemistry, The University of Hong Kong, Pofkulam Road, Hong Kong, China \\ ${ }^{2}$ Department of Chemistry, The Hong Kong University of Science and Technology, Hong Kong, China \\ ${ }^{3}$ Hefei National Laboratory for Physical Sciences at the Microscale, University of Science and Technology \\ of China, Hefei 230026, China
}

(Received 20 April 2012; accepted 3 July 2012; published online 27 July 2012)

\begin{abstract}
Basing on our hierarchical equations of motion for time-dependent quantum transport [X. Zheng, G. H. Chen, Y. Mo, S. K. Koo, H. Tian, C. Y. Yam, and Y. J. Yan, J. Chem. Phys. 133, 114101 (2010)], we develop an efficient and accurate numerical algorithm to solve the Liouville-von-Neumann equation. We solve the real-time evolution of the reduced single-electron density matrix at the tightbinding level. Calculations are carried out to simulate the transient current through a linear chain of atoms, with each represented by a single orbital. The self-energy matrix is expanded in terms of multiple Lorentzian functions, and the Fermi distribution function is evaluated via the Padè spectrum decomposition. This Lorentzian-Padè decomposition scheme is employed to simulate the transient current. With sufficient Lorentzian functions used to fit the self-energy matrices, we show that the lead spectral function and the dynamics response can be treated accurately. Compared to the conventional master equation approaches, our method is much more efficient as the computational time scales cubically with the system size and linearly with the simulation time. As a result, the simulations of the transient currents through systems containing up to one hundred of atoms have been carried out. As density functional theory is also an effective one-particle theory, the Lorentzian-Padè decomposition scheme developed here can be generalized for first-principles simulation of realistic systems. (C) 2012 American Institute of Physics. [http://dx.doi.org/10.1063/1.4737864]
\end{abstract}

\section{INTRODUCTION}

Quantum transport has been a focus of research interests due to the rapid developments of nanotechnology and semiconductor industry. ${ }^{1}$ Time-dependent quantum transport is an important subject of study. On experimental side, recent advancement has been made for the transient current measurement of quantum dots ${ }^{2}$ and coherent manipulation of charge. ${ }^{3}$ On the theoretical side, the Keldysh non-equilibrium Green's function (NEGF) method has been employed often to calculate the steady state currents ${ }^{4-6}$ and the transient ${ }^{7,8}$ currents through nanoscopic devices. Based on the timedependent density-functional theory (TDDFT), the master equation approaches have been developed to calculate transient current. ${ }^{9-14}$

In particular, the NEGF formalism was combined with TDDFT, and the resulting TDDFT-NEGF formalism has been developed to simulate the transient charge transport through molecular and nano-devices. ${ }^{12,15-22}$ We note that there are debates on the fundamentals of TDDFT. ${ }^{23,24}$ Other methods have been proposed to study the transient process. For example, Stefanouchi, Kurth, Gross, and others have used the wave function propagator scheme with the transparent boundary conditions (TBC) to calculate the electron evolutions. ${ }^{25-27}$ The TBC was developed in 1980s to mimic the infinite environment by correcting wave functions at the boundary, but

\footnotetext{
a)ghc@everest.hku.hk.
}

with the memory term to record the quantities in the earlier time domain.

In the NEGF calculations, the wide band limit (WBL) approximation is widely used to simplify the convolution calculation of self-energy. ${ }^{7,12}$ This approximation, however, cannot properly mimic the realistic energy spectra of the leads, and may thus lose key information and results in improper transient current. Tanimura and Kubo developed the hierarchical equations of motion (HEOM) for bosonic open systems. ${ }^{28}$ Yan et al. have extended the HEOM method to Fermionic system by using reduced many-electron density matrix (RMDM) or RMDM-HEOM. ${ }^{29}$ Similar to the other master equation approaches, the RMDM-HEOM method is computationally very expensive, and limited to the model systems with few orbitals. Recently, we have developed a firstprinciple HEOM for the reduced single-electron density matrix (RSDM) (denoted as RSDM-HEOM) ${ }^{30}$ It turns out that the RSDM-HEOM is in principle exact by just including the first- and second-tier auxiliary density matrices (ADM) within the framework of TDDFT-NEGF theory ${ }^{29,30}$ and goes thus beyond the WBL approximation. As the RSDM is much simpler than the RMDM, the RSDM-HEOM method is expected to be much more efficient so that it may be used to simulate the realistic systems.

This paper continues our recent effort on RSDM-HEOM formalism. ${ }^{30}$ A complete set of spectrum coefficients are derived for numerical implementation by fitting the selfenergy matrix with a multi-Lorentzian expansion scheme and 
evaluating the Fermi distribution function via the Padè spectrum decomposition. The tight-binding (TB) model is employed to model a chain of atoms with each atom described by a single energy level. The new Padè spectrum decomposition reduces the expansion terms with better accuracy. ${ }^{31}$

The paper is organized as follows. In Sec. II, a demonstration is given for the RSDM-HEOM theory, specially for the spectral decomposition method for the self-energy; and the central equations of the RSDM-HEOM theory are given. In Sec III, we describe the numerical implementation of the RSDM-HEOM method, in particular, the detailed Lorentzian fitting scheme for the self-energy matrix. In Sec. IV, we apply our method to simulate the transient currents through the two- and four-atom systems employing the TB models. Different Lorentzian fitting schemes are compared. In Sec. V, we examine the numerical efficiency by carrying out the simulation on the systems containing up to one hundred of atoms. Summary is given in Sec. VI.

\section{THEORY}

\section{A. Hierarchical equation of motions for reduced single-electron density matrix}

The many-electron density operator can be projected onto the Liouville space made of pairs of basis functions, and results in the RSDM. Its EOM, derived from Schrodinger equation, is

$$
i \dot{\sigma}(t)=[\mathbf{h}(t), \boldsymbol{\sigma}(t)],
$$

where $\sigma(t)$ is the RSDM, $\mathbf{h}(t)$ is the Hamiltonian or Fock operator of the system, and the square bracket denotes a commutator. The atomic unit is used throughout the paper.

Let us consider a two-terminal device with left and right electrodes. The density matrix and Hamiltonian matrix are partitioned and the corresponding EOM is written as ${ }^{12}$

$$
i \dot{\sigma}_{D}(t)=\left[\mathbf{h}_{D}(t), \sigma_{D}(t)\right]+\sum_{\alpha=L, R}\left(\mathbf{h}_{D \alpha} \boldsymbol{\sigma}_{\alpha D}-\boldsymbol{\sigma}_{D \alpha} \mathbf{h}_{\alpha D}\right),
$$

where $L, R$, and $D$ denote the left lead, right lead, and device, respectively. $\mathbf{h}_{D}$ is the device Hamiltonian, $\mathbf{h}_{D \alpha}$ is the coupling Hamiltonian between the device $(D)$ and lead $\alpha$ ( $\alpha$ denotes $L$ or $R$ ), and similarly $\sigma_{D}$ and $\sigma_{\alpha D}$ are defined.

This equation can be rewritten as the following differential-integral equation using the Langreth rule: ${ }^{7,12}$

$$
\begin{aligned}
i \dot{\sigma}_{D}(t)= & {\left[\mathbf{h}_{D}(t), \sigma_{D}(t)\right]+i \int_{-\infty}^{t} d \tau\left[\mathbf{G}_{D}^{<}(t, \tau) \cdot \boldsymbol{\Sigma}_{\alpha}^{>}(\tau, t)\right.} \\
& \left.-\mathbf{G}_{D}^{>}(t, \tau) \cdot \boldsymbol{\Sigma}_{\alpha}^{<}(\tau, t)-\text { H.c. }\right],
\end{aligned}
$$

where $\Sigma_{\alpha}^{x}(t, \tau)$ are the lesser $(\mathrm{x}=<)$ or greater $(\mathrm{x}=>)$ selfenergy; $\mathbf{G}_{D}^{x}(t, \tau)$ are the lesser or greater Green's function of device. H.c. means the Hermitian conjugate.

It is difficult to solve this equation directly, since other quantities like $\mathbf{G}_{D}^{<}(t, \tau)$ and $\boldsymbol{\Sigma}_{\alpha}^{>}(\tau, t)$ need to be calculated separately and $\mathbf{G}_{D}^{<,>}(t, \tau)$ evolution results in the two-time integral. ${ }^{7,12}$ However, according to Ref. 30, if we introduce the energy-resolved self-energies $\boldsymbol{\Sigma}_{\alpha}^{<,>}(\varepsilon, \tau, t)$, $\left(\Sigma_{\alpha}^{<,>}(\tau, t)=\int d \varepsilon \cdot \Sigma_{\alpha}^{<,>}(\varepsilon, \tau, t)\right)$, and the following auxiliary density matrices of the first- and second-tier:

$$
\begin{gathered}
\boldsymbol{\varphi}_{\alpha}(\varepsilon, t)=i \int_{-\infty}^{t} d \tau\left[\mathbf{G}_{D}^{<}(t, \tau) \cdot \mathbf{\Sigma}_{\alpha}^{>}(\varepsilon, \tau, t)\right. \\
\left.-\mathbf{G}_{D}^{>}(t, \tau) \cdot \boldsymbol{\Sigma}_{\alpha}^{<}(\varepsilon, \tau, t)\right], \\
\boldsymbol{\varphi}_{\alpha \alpha^{\prime}}\left(\varepsilon, \varepsilon^{\prime}, t\right)=i \int_{-\infty}^{t} d t_{1} \int_{-\infty}^{t} d t_{2}\left\{\left[\boldsymbol{\Sigma}_{\alpha^{\prime}}^{<}\left(\varepsilon^{\prime}, t, t_{1}\right) \cdot \mathbf{G}_{D}^{a}\left(t_{1}, t_{2}\right)\right.\right. \\
\left.+\boldsymbol{\Sigma}_{\alpha^{\prime}}^{r}\left(\varepsilon^{\prime}, t, t_{1}\right) \cdot \mathbf{G}_{D}^{<}\left(t_{1}, t_{2}\right)\right] \boldsymbol{\Sigma}_{\alpha}^{>}\left(\varepsilon, t_{2}, t\right) \\
-\left[\boldsymbol{\Sigma}_{\alpha^{\prime}}^{>}\left(\varepsilon^{\prime}, t, t_{1}\right) \cdot \mathbf{G}_{D}^{a}\left(t_{1}, t_{2}\right)\right. \\
\left.\left.+\boldsymbol{\Sigma}_{\alpha^{\prime}}^{r}\left(\varepsilon^{\prime}, t, t_{1}\right) \cdot \mathbf{G}_{D}^{>}\left(t_{1}, t_{2}\right)\right] \mathbf{\Sigma}_{\alpha}^{<}\left(\varepsilon, t_{2}, t\right)\right\},
\end{gathered}
$$

we derive the following set of differential-integral equations:

$i \dot{\sigma}_{D}(t)=\left[\mathbf{h}_{D}(t), \sigma_{D}(t)\right]-\sum_{\alpha}^{N_{\alpha}} \int d \varepsilon \cdot\left[\boldsymbol{\varphi}_{\alpha}(\varepsilon, t)-\boldsymbol{\varphi}_{\alpha}^{\dagger}(\varepsilon, t)\right]$,

$$
\begin{aligned}
i \dot{\boldsymbol{\varphi}}_{\alpha}(\varepsilon, t)= & {\left[\mathbf{h}_{D}(t)-\varepsilon-\Delta_{\alpha}(t)\right] \cdot \boldsymbol{\varphi}_{\alpha}(\varepsilon, t) } \\
& +\left[f_{\alpha}(\varepsilon)-\sigma_{D}(t)\right] \boldsymbol{\Lambda}_{\alpha}(\varepsilon) \\
& +\sum_{\alpha^{\prime}}^{N_{\alpha}} \int d \varepsilon^{\prime} \boldsymbol{\varphi}_{\alpha \alpha^{\prime}}\left(\varepsilon, \varepsilon^{\prime}, t\right),
\end{aligned}
$$

$$
\begin{aligned}
i \dot{\boldsymbol{\varphi}}_{\alpha, \alpha^{\prime}}\left(\varepsilon, \varepsilon^{\prime}, t\right)= & -\left[\varepsilon+\Delta_{\alpha}(t)-\varepsilon^{\prime}-\Delta_{\alpha^{\prime}}(t)\right] \cdot \boldsymbol{\varphi}_{\alpha, \alpha^{\prime}}\left(\varepsilon, \varepsilon^{\prime}, t\right) \\
& +\boldsymbol{\Lambda}_{\alpha^{\prime}}\left(\varepsilon^{\prime}\right) \cdot \boldsymbol{\varphi}_{\alpha}(\varepsilon, t)-\boldsymbol{\varphi}_{\alpha^{\prime}}^{\dagger}\left(\varepsilon^{\prime}, t\right) \cdot \boldsymbol{\Lambda}_{\alpha}(\varepsilon),
\end{aligned}
$$

where $\boldsymbol{\Lambda}_{\alpha}(\varepsilon)$ is the linewidth function, and $f_{\alpha}(\varepsilon)$ is the Fermi function for lead $\alpha, f_{\alpha}(\varepsilon)=1 /\left(1+\exp \left[\beta\left(\varepsilon-\mu_{\alpha}\right)\right]\right), \beta$ $=1 / k_{B} T$, is the reciprocal temperature, and $\mu_{\alpha}$ is the chemical potential of lead $\alpha . \Delta_{\alpha}(t)$ is the time-dependent bias potential in lead $\alpha$.

This is the RSDM-HEOM. The derivation of Eqs. (5)-(7) based on the NEGF formalism is given in the Appendix. In the framework of TDDFT, we note that the hierarchy exactly terminates at the second-tier ADM.

In practical HEOM calculations, it is computationally expansive to solve these differential-integral equations. Using the residue theorem, we may expand the self-energy into a summation, and each term in the summation can be expressed as a time exponential term, (see details in Sec. II B). The integral in the HEOMs may also be changed into a summation. The discrete version of HEOM is thus resulted, as a set of homogenous differential equations (see the Appendix for details), which is numerically tractable, even for the firstprinciples calculations.

\section{B. Self-energy decomposition method}

From the Appendix, the steady state self-energy is expressed in the Fourier integral form, and the integral can be extended to the contour integral in the complex plane. For 
example, $\widetilde{\boldsymbol{\Sigma}}_{\alpha}^{<}(\tau-t)$ can be expressed as

$$
\begin{aligned}
\widetilde{\mathbf{\Sigma}}_{\alpha}^{<}(\tau-t) & =\frac{i}{2 \pi} \int_{-\infty}^{+\infty} f_{\alpha}(\varepsilon) \boldsymbol{\Lambda}_{\alpha}(\varepsilon) \cdot e^{-i \varepsilon(\tau-t)} d \varepsilon \\
& =\frac{i}{2 \pi} \oint f_{\alpha}(z) \boldsymbol{\Lambda}_{\alpha}(z) \cdot e^{-i z(\tau-t)} d z .
\end{aligned}
$$

Here, the closed contour consists of the real axis and a semicircle $C_{R}$ in the upper or lower half plane at infinity, as shown in Fig. 1. The analytic continuation of $f_{\alpha}(\varepsilon), \boldsymbol{\Lambda}_{\alpha}(\varepsilon)$ and $e^{-i \varepsilon(\tau-t)}$ into complex plane is needed, in which the complex variable $z$ replaces the real variable $\varepsilon$.

The last equality of Eq. (8) exists only when the integral on $C_{R}$ is zero. With the Cauchy's residue theorem, ${ }^{32}$ the self-energy can be transformed into the summation form. This technique is called the self-energy decomposition method.
To ensure the integral in Eq. (8) decays on the semicircle path $C_{R}$, besides $f_{\alpha}(z) \boldsymbol{\Lambda}_{\alpha}(z)$ decays to zero (see their expansions later), the exponential term $e^{-i z(\tau-t)}$ must decay to zero on $C_{R}$ as well. Therefore, for different signs of $\tau-t$, two different $C_{R}$ should be chosen, as shown in Fig. 1 .

(a) When $\tau-t<0, z$ must be in the upper contour $C_{R}^{+}$to ensure that $e^{-i z(\tau-t)}$ decays to zero as $C_{R}^{+}$expands to infinity (i.e., if $z$ is on the positive imaginary axis, we have $z=i R(\mathrm{R}$ $\rightarrow+\infty)$ and $\exp [-i z(\tau-t)]=\exp [-R|t-\tau|] \rightarrow 0)$. Thus, the residue theorem is used to transform the integral in Eq. (8) into a summation

$$
\tilde{\mathbf{\Sigma}}_{\alpha}^{<,>}(\tau-t)=\sum_{k}^{N_{k}} \mathbf{A}_{\alpha k}^{<,>+} \cdot e^{-\gamma_{\alpha k}^{+}(t-\tau)}, \quad(\tau-t<0) .
$$

$\mathbf{A}_{\alpha k}^{<,>+}$and $\gamma_{\alpha k}^{+}$result from the residue calculations, as shown below

$$
\begin{gathered}
\mathbf{A}_{\alpha k}^{<+}=\left\{\begin{array}{ll}
\frac{i \eta_{d}}{2 W_{d}} f_{\alpha}\left(\Omega_{d}+\mu_{\alpha}+i W_{d}\right) \overline{\boldsymbol{\Lambda}}_{\alpha k}, & \left(1 \leq k \leq N_{d}\right) \\
-\frac{R_{p}}{\beta} \sum_{d=1}^{N_{d}} \frac{\eta_{d}}{\left(x_{p}^{+}-\Omega_{d}-\mu_{\alpha}\right)^{2}+W_{d}^{2}} \overline{\mathbf{\Lambda}}_{\alpha d}, & \left(N_{d}<\mathrm{k} \leq N_{k}\right)
\end{array},\right. \\
\mathbf{A}_{\alpha k}^{>+}=\left\{\begin{array}{ll}
\frac{-i \eta_{d}}{2 W_{d}}\left[1-f_{\alpha}\left(\Omega_{d}+\mu_{\alpha}+i W_{d}\right)\right] \overline{\boldsymbol{\Lambda}}_{\alpha k}, & \left(1 \leq k \leq N_{d}\right) \\
-\frac{R_{p}}{\beta} \sum_{d=1}^{N_{d}} \frac{\eta_{d}}{\left(x_{p}^{+}-\Omega_{d}-\mu_{\alpha}\right)^{2}+W_{d}^{2}} \overline{\mathbf{\Lambda}}_{\alpha d}, & \left(N_{d}<\mathrm{k} \leq N_{k}\right)
\end{array},\right. \\
\gamma_{\alpha k}^{+}= \begin{cases}-i\left(\Omega_{k}+\mu_{\alpha}+i W_{k}\right), & \left(1 \leq k \leq N_{d}\right) \\
-i\left(\mu_{\alpha}+z_{p}^{+} / \beta\right), & \left(\mathrm{N}_{d}<k \leq N_{k}\right)\end{cases}
\end{gathered}
$$

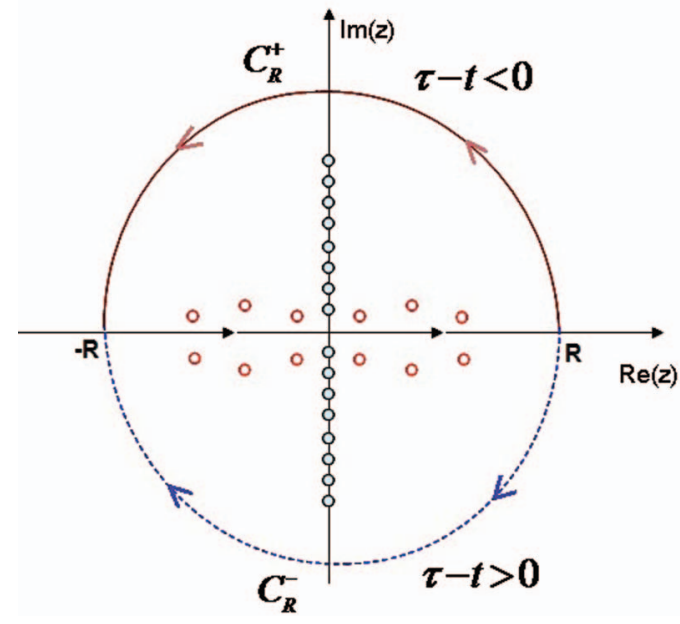

FIG. 1. Contours for the integral of linewidth function. The upper contour (solid line, denoted as $C_{R}^{+}$) and lower contour (dotted line, denoted as $C_{R}^{-}$) are used for different signs of $\tau-t$. The filled circles (on y axis) and hollow circles represent the Padè poles $\left(\mu_{\alpha}=0\right)$ and Lorentzian poles, respectively. The arrows indicate the contour integral directions. where $x_{p}^{+}=\mu_{\alpha}+z_{p}^{+} / \beta$, and $p=k-N_{d}$. " + " means the residues come from the upper integral contour. The definitions of $N_{d}, \eta_{d}, W_{d}, \Omega_{d}, \bar{\Lambda}_{\alpha k}, R_{p}$, and $z_{p}^{+}$are derived from the expansions of $\boldsymbol{\Lambda}_{\alpha}(\varepsilon)$ and $f_{\alpha}(z)$, which will be detailed later.

This integral-summation transformation on the upper contour (see also Eq. (A12) or Eq. (A13) in the Appendix) is used for $\boldsymbol{\Sigma}_{\alpha k}^{<,>}(\tau, t)$ in $\boldsymbol{\varphi}_{\alpha k}(t)$, or for $\boldsymbol{\Sigma}_{\alpha k}^{<,>}\left(t_{2}, t\right)$ in $\boldsymbol{\varphi}_{\alpha k, \alpha^{\prime} k^{\prime}}(t)$, since from Eqs. (3) and (4), it is apparent that in $\boldsymbol{\Sigma}_{\alpha}^{<,>}(\varepsilon, \tau, t)\left(\right.$ or $\left.\boldsymbol{\Sigma}_{\alpha k}^{<,>}(\tau, t)\right), \tau-t<0$ and in $\boldsymbol{\Sigma}_{\alpha}^{<,>}\left(\varepsilon, t_{2}, t\right)$ (or $\left.\Sigma_{\alpha k}^{<,>}\left(t_{2}, t\right)\right), t_{2}-t<0$.

(b) When $\tau-t>0, z$ must be in the lower contour $C_{R}^{-}$ to ensure that $e^{-i z(\tau-t)}$ decays to zero. Thus, the integral in Eq. (8) is changed into a summation

$$
\widetilde{\mathbf{\Sigma}}_{\alpha}^{<,>}(\tau-t)=\sum_{k}^{N_{k}} \mathbf{A}_{\alpha k}^{<,>-} \cdot e^{+\gamma_{\alpha k}^{-}(t-\tau)}, \quad(\tau-t>0),
$$


where

$$
\begin{gathered}
\mathbf{A}_{\alpha k}^{<-}= \begin{cases}\frac{i \eta_{d}}{2 W_{d}} f_{\alpha}\left(\Omega_{d}+\mu_{\alpha}-i W_{d}\right) \overline{\boldsymbol{\Lambda}}_{\alpha k}, & \left(1 \leq k \leq N_{d}\right) \\
\frac{R_{p}}{\beta} \sum_{d=1}^{N_{d}} \frac{\eta_{d}}{\left(x_{p}^{-}-\Omega_{d}-\mu_{\alpha}\right)^{2}+W_{d}^{2}} \overline{\mathbf{\Lambda}}_{\alpha d}, \quad\left(N_{d}<\mathrm{k} \leq N_{k}\right)\end{cases} \\
\mathbf{A}_{\alpha k}^{>-}= \begin{cases}\frac{-i \eta_{d}}{2 W_{d}}\left[1-f_{\alpha}\left(\Omega_{d}+\mu_{\alpha}-i W_{d}\right)\right] \overline{\boldsymbol{\Lambda}}_{\alpha k}, & \left(1 \leq k \leq N_{d}\right) \\
\frac{R_{p}}{\beta} \sum_{d=1}^{N_{d}} \frac{\eta_{d}}{\left(x_{p}^{-}-\Omega_{d}-\mu_{\alpha}\right)^{2}+W_{d}^{2}} \overline{\mathbf{\Lambda}}_{\alpha d}, \quad\left(N_{d}<k \leq N_{k}\right)\end{cases} \\
\gamma_{\alpha k}^{-}= \begin{cases}i\left(\Omega_{k}+\mu_{\alpha}-i W_{k}\right), & \left(1 \leq k \leq N_{d}\right) \\
i\left(\mu_{\alpha}+z_{p}^{-} / \beta\right), & \left(\mathrm{N}_{d}<k \leq N_{k}\right)\end{cases}
\end{gathered}
$$

and $x_{p}^{-}=\mu_{\alpha}+z_{p}^{-} / \beta$, and $p=k-N_{d}$. "-" denotes the lower integral contour.

This integral-summation transformation on the lower contour (see Eq. (A13) in the Appendix) is used for $\boldsymbol{\Sigma}_{\alpha^{\prime} k^{\prime}}^{<,>}\left(t, t_{1}\right)$ in $\boldsymbol{\varphi}_{\alpha k, \alpha^{\prime} k^{\prime}}(t)$, as $t-t_{1}>0$ in $\boldsymbol{\Sigma}_{\alpha^{\prime}}^{<,>}\left(\varepsilon^{\prime}, t, t_{1}\right)$ (or $\left.\Sigma_{\alpha^{\prime} k^{\prime}}^{<,>}\left(t, t_{1}\right)\right)$.

The linewidth matrices $\boldsymbol{\Lambda}_{\alpha}(\varepsilon)$ are expanded in the multiple Lorentzian terms as follows:

$$
\boldsymbol{\Lambda}_{\alpha}(\varepsilon) \approx \sum_{d=1}^{N_{d}} \frac{\eta_{d}}{\left(\varepsilon-\Omega_{d}\right)^{2}+W_{d}^{2}} \overline{\boldsymbol{\Lambda}}_{\alpha d}
$$

where $\Omega_{d}, W_{d}, \eta_{d}$ are the central position, width, and amplitude (scalar part) for the $d$ th Lorentzian function, respectively. $N_{d}$ is the number of Lorentzian pole pairs. This Lorentzian expression has the poles $\left(\Omega_{d}+\mu_{\alpha}+i W_{d}\right)$ in the upper and the poles $\left(\Omega_{d}+\mu_{\alpha}-i W_{d}\right)$ in the lower complex plane, as shown in Fig. 1. Section III A shows how the matrix part $\overline{\boldsymbol{\Lambda}}_{\alpha d}$ is obtained from the surface Green's functions and coupling matrices.

In our approach, the Padè spectrum decomposition ${ }^{31}$ is used to expand the Fermi-Dirac function as follows:

$$
\begin{aligned}
f_{\alpha}(z)=\frac{1}{1+\exp (z)} & =\frac{1}{2}-\frac{1}{2} \frac{\sinh (z)}{\cosh (z)} \\
& \approx \frac{1}{2}+\sum_{p=1}^{N_{p}}\left(\frac{R_{p}}{z-z_{p}^{+}}+\frac{R_{p}}{z-z_{p}^{-}}\right)
\end{aligned}
$$

where $z=\beta\left(\varepsilon-\mu_{\alpha}\right), R_{p}$ is the $p$ th residue in the Padè spectrum decomposition, and $z_{p}^{ \pm}$is the $p$ th Padè pole in the upper $(+)$ or lower (-) complex plane, $N_{p}$ is the number of Padè pole pairs.

We restate that in the calculations of $\mathbf{A}_{\alpha k}^{<,> \pm}$and $\gamma_{\alpha k}^{ \pm}$, the Lorentzian and Padè poles with positive imaginary part are chosen for the upper contour case; and the poles with the negative imaginary part are chosen for the lower contour case.

\section{The central equations of the HEOM method}

Substituting Eqs. (9)-(12) into Eqs. (3)-(7) (details are given in the Appendix), we obtain the following central equations of our RSDM-HEOM formalism:

$$
\begin{aligned}
i \dot{\boldsymbol{\sigma}}(t)= & {\left[\mathbf{h}_{D}(t), \boldsymbol{\sigma}(t)\right]-\sum_{\alpha}^{N_{\alpha}} \sum_{k=1}^{N_{k}}\left(\boldsymbol{\varphi}_{\alpha k}(t)-\boldsymbol{\varphi}_{\alpha k}^{\dagger}(t)\right), } \\
i \dot{\boldsymbol{\varphi}}_{\alpha k}(t)=\left[\mathbf{h}_{D}(t)-i \gamma_{\alpha k}^{+}-\boldsymbol{\Delta}_{\alpha}(t)\right] \boldsymbol{\varphi}_{\alpha k}(t)-i\left[\boldsymbol{\sigma}(t) \mathbf{A}_{\alpha k}^{>+}\right. & \\
& \left.+\overline{\boldsymbol{\sigma}}(t) \mathbf{A}_{\alpha k}^{<+}\right]+\sum_{\alpha^{\prime}}^{N_{\alpha}} \sum_{k^{\prime}=1}^{N_{k}} \boldsymbol{\varphi}_{\alpha k, \alpha^{\prime} k^{\prime}}(t)
\end{aligned}
$$

$$
\begin{aligned}
i \dot{\varphi}_{\alpha k, \alpha^{\prime} k^{\prime}}(t)= & -\left[i \gamma_{\alpha k}^{+}+\boldsymbol{\Delta}_{\alpha}(t)+i \gamma_{\alpha^{\prime} k^{\prime}}^{-}-\Delta_{\alpha^{\prime}}(t)\right] \cdot \boldsymbol{\varphi}_{\alpha k, \alpha^{\prime} k^{\prime}}(t) \\
& +i\left(\mathbf{A}_{\alpha^{\prime} k^{\prime}}^{>-}-\mathbf{A}_{\alpha^{\prime} k^{\prime}}^{<-}\right) \boldsymbol{\varphi}_{\alpha k}(t) \\
& -i \boldsymbol{\varphi}_{\alpha^{\prime} k^{\prime}}^{\dagger}(t)\left(\mathbf{A}_{\alpha k}^{>+}-\mathbf{A}_{\alpha k}^{<+}\right)
\end{aligned}
$$

where $\overline{\boldsymbol{\sigma}}(t)=\mathbf{I}-\boldsymbol{\sigma}(t)$ and $N_{k}=N_{d}+N_{p}$ are the total number of the Padè and Lorentzian poles in the contour integral. We term Eqs. (15)-(17) and their solutions as the Lorentzian-Padè decomposition scheme.

The more $N_{k}$ is, the more accurate the results are. However, the large value of $N_{k}$ implies that more coupled equations are to be solved, and thus more computational time is required. The key to the success of our approach is to have a reasonable value of $N_{k}$ that leads to the desirable accuracy. The number of the Padè poles is determined by the simulation temperature and energy intervals, while the number of Lorentzian poles is determined by the complexity of self-energy matrices. In Sec. III A below, we describe the Lorentzian expansion for the self-energy matrices.

\section{NUMERICAL IMPLEMENTATION}

\section{A. Matrix-based Lorentzian expansion}

Lorentzian expansion of the linewidth (or spectral) function is widely employed in quantum dissipation theory. The linewidth function is usually a scalar function. However, in 
our case, it is a matrix. We need to develop a new Lorentzian expansion scheme here.

The linewidth matrix $\boldsymbol{\Lambda}_{\alpha}(\varepsilon)$ is related to the self-energy, or the lead Green's function,

$$
\Lambda_{\alpha, i j}(\varepsilon)=\sum_{k_{1}, k_{2}}^{N_{i j}} h_{i k_{1}} \cdot \widetilde{g}_{\alpha, k_{1} k_{2}}(\varepsilon) \cdot h_{k_{2} j},
$$

where $h_{i j}$ is the coupling matrix between the lead and device; $N_{i j}$ is the upper bound of the summation, which is determined by nonzero coupling matrix $h_{i j}$ [see Eq. (22) below]; and $\widetilde{g}_{\alpha, k_{1} k_{2}}$ is related to the lead Green's function, and it can be approximated by the Lorentzian expansion,

$$
\widetilde{g}_{\alpha, k_{1} k_{2}}(\varepsilon)=-2 \operatorname{Im}\left[g_{\alpha, k_{1} k_{2}}^{r}(\varepsilon)\right]=\sum_{d=1}^{N_{k_{1} k_{2}}} L_{\alpha, k_{1} k_{2}}(\varepsilon, d),
$$

where $L_{\alpha, k_{1} k_{2}}(\varepsilon, d)$ is the $d$ th Lorentzian expansion term, as shown in Eq. (13). The subscripts denote this expansion only for $\widetilde{g}_{\alpha, k_{1} k_{2}}$, and $N_{k_{1} k_{2}}$ is the number of expansion terms, which is determined by the required accuracy.

We can thus rewrite $\boldsymbol{\Lambda}_{\alpha}(\varepsilon)$ as a general expansion in the following derivation:

$$
\begin{aligned}
\Lambda_{\alpha, i j}(\varepsilon) & =\sum_{k_{1}, k_{2}}^{N_{i j}} h_{i k_{1}} \cdot h_{k_{2} j} \cdot \sum_{d=1}^{N_{k_{1} k_{2}}} L_{\alpha, k_{1} k_{2}}(\varepsilon, d) \\
& =\sum_{k_{1}, k_{2}}^{N_{i j}} h_{i k_{1}} \cdot h_{k_{2} j} \cdot \sum_{d_{0}=1}^{N_{d}} \lambda\left(\alpha, k_{1}, k_{2}, d_{0}\right) L\left(\varepsilon, d_{0}\right),
\end{aligned}
$$

where $N_{d}$ is the total number of the Lorentzian functions used for $\boldsymbol{\Lambda}_{\alpha}(\varepsilon)$, and $\lambda\left(\alpha, k_{1}, k_{2}, d_{0}\right)$ is the weight factor. In the last equality of Eq. (20), a global index $d_{0}$ is used to replace the local (specified for each $\widetilde{g}_{\alpha, k_{1} k_{2}}$ ) index $\left(\alpha, k_{1}, k_{2}, d\right)$; and the weight factor is used to distinguish which Lorentzians $\left(L_{\alpha, k_{1} k_{2}}(\varepsilon, d)\right.$ or $\left.L\left(\varepsilon, d_{0}\right)\right)$ are needed in the summation for $\widetilde{g}_{\alpha, k_{1} k_{2}}$. We further write out

$$
\Lambda_{\alpha, i j}(\varepsilon)=\sum_{d_{0}}^{N_{d}} L\left(\varepsilon, d_{0}\right) \cdot \bar{\Lambda}_{\alpha d_{0}, i j}
$$

where

$$
\bar{\Lambda}_{\alpha d_{0}, i j}=\sum_{k_{1}, k_{2}}^{N_{i j}} h_{i, k_{1}} \cdot h_{k_{2}, j} \cdot \lambda\left(\alpha, k_{1}, k_{2}, d_{0}\right) .
$$

This formula is the detailed form of Eq. (13).

It is emphasized that in practice the coupling matrix is often a sparse matrix, and the element $h_{i j}$ may be set to zero once its value is below some cut-off threshold. This reduces the number of the nonzero elements of the linewidth matrix, and thus reduces the computational efforts. In the generalized tight-binding models, the coupling matrix can be expressed as

$$
h_{i j}=\left\{\begin{array}{cc}
h_{i j} & \left(|i-j| \leq m_{0}\right) \\
0 & \left(|i-j|>m_{0}\right)
\end{array},\right.
$$

where $m_{0}$ is the order for how nearest the neighbor interactions are considered. For the nearest neighbor model of 1D system, $m_{0}=1$; and for the next nearest neighbor model, $m_{0}=2$.

\section{B. Initial values and numerical integration in time domain}

To integrate the HEOM in time domain, we need to know the initial values of $\sigma, \varphi_{\alpha k}$, and $\varphi_{\alpha k \alpha^{\prime} k^{\prime}}$, or the steady solutions of Eqs. (15)-(17). For these steady state solutions, the time derivatives of the three sets of density matrices are zero [in Eqs. (15)-(17), the left hand sides are set to zero]. Equations (15) and (17) include complex $\boldsymbol{\varphi}_{\alpha k}$ and $\boldsymbol{\varphi}_{\alpha k}^{\dagger}$, and we have to separate these density matrices into the real and imaginary part, like $\sigma=\sigma^{R}+i \cdot \sigma^{I}$ and $\varphi_{\alpha k}=\varphi_{\alpha k}^{R}+i \cdot \varphi_{\alpha k}^{I}$ etc, and regarding them as real unknown variables. Then all other quantities in Eqs. (15)-(17) are decomposed into the real and imaginary part as well, to reach the following set of equations:

$$
\left[\mathbf{h}_{D}(t), \boldsymbol{\sigma}(t)\right]-\sum_{\alpha}^{N_{\alpha}} \sum_{k=1}^{N_{k}}\left(\boldsymbol{\varphi}_{\alpha k}(t)-\boldsymbol{\varphi}_{\alpha k}^{\dagger}(t)\right)=0
$$

$$
\begin{aligned}
& {\left[\mathbf{h}_{D}(t)-i \gamma_{\alpha k}^{+}-\boldsymbol{\Delta}_{\alpha}(t)\right] \boldsymbol{\varphi}_{\alpha k}(t)-i\left[\boldsymbol{\sigma}(t) \mathbf{A}_{\alpha k}^{>+}+\overline{\boldsymbol{\sigma}}(t) \mathbf{A}_{\alpha k}^{<+}\right]} \\
& \quad+\sum_{\alpha^{\prime}}^{N_{\alpha}} \sum_{k^{\prime}=1}^{N_{k}} \boldsymbol{\varphi}_{\alpha k, \alpha^{\prime} k^{\prime}}(t)=0 \\
& -\left[i \gamma_{\alpha k}^{+}+\boldsymbol{\Delta}_{\alpha}(t)+i \gamma_{\alpha^{\prime} k^{\prime}}^{-}-\boldsymbol{\Delta}_{\alpha^{\prime}}(t)\right] \cdot \boldsymbol{\varphi}_{\alpha k, \alpha^{\prime} k^{\prime}}(t) \\
& +i\left(\mathbf{A}_{\alpha^{\prime} k^{\prime}}^{>-}-\mathbf{A}_{\alpha^{\prime} k^{\prime}}^{<-}\right) \boldsymbol{\varphi}_{\alpha k}(t)-i \boldsymbol{\varphi}_{\alpha^{\prime} k^{\prime}}^{\dagger}(t)\left(\mathbf{A}_{\alpha k}^{>+}-\mathbf{A}_{\alpha k}^{<+}\right)=0
\end{aligned}
$$

In the equations above, the real and imaginary parts are set to zero. After rearranging the above equations into a large matrix equation: $\mathbf{A} \cdot \mathbf{x}=\mathbf{B}$, we may use the direct or iterative method to solve it and to get the solution. Then the initial values of $\sigma_{i j}, \varphi_{\alpha k, i j}$, and $\varphi_{\alpha k, \alpha^{\prime} k^{\prime}, i j}$ are recovered from this solution.

As the initial values are determined, the fourth-order Runge-Kutta scheme is employed to propagate the evolution of $\boldsymbol{\sigma}, \boldsymbol{\varphi}_{\alpha k}$, and $\boldsymbol{\varphi}_{\alpha k, \alpha^{\prime} k^{\prime}}$ in Eqs. (15)-(17). The time-dependent current of the system is given by the following formula: ${ }^{30}$

$$
\mathbf{J}_{\alpha}(t)=-\int d \mathbf{r} \frac{\partial}{\partial t} \rho(\mathbf{r}, t)=-\operatorname{tr}\left[\mathbf{Q}_{\alpha}(t)\right] .
$$

In the first-principles calculations, the current density may be redefined by adding the contribution of the nonlocal potential, to conserve the current. ${ }^{33}$

\section{RESULTS}

\section{A. Numerical test on a single level system}

To demonstrate the validity of our method, we calculate the transient current through a single site, and compare our calculation with the exact numerical values in Ref. 34. The device region consists of a single state with on-site energy $\left(\varepsilon_{0}(t)=\varepsilon_{0}+\Delta(t)\right)$ and is coupled to two leads. The bias voltage is applied to shift the energy levels of the leads: $\varepsilon_{k \alpha}(t)$ $=\varepsilon_{k \alpha}+\Delta_{\alpha}(t)$. The energy spectrum of the lead is described 

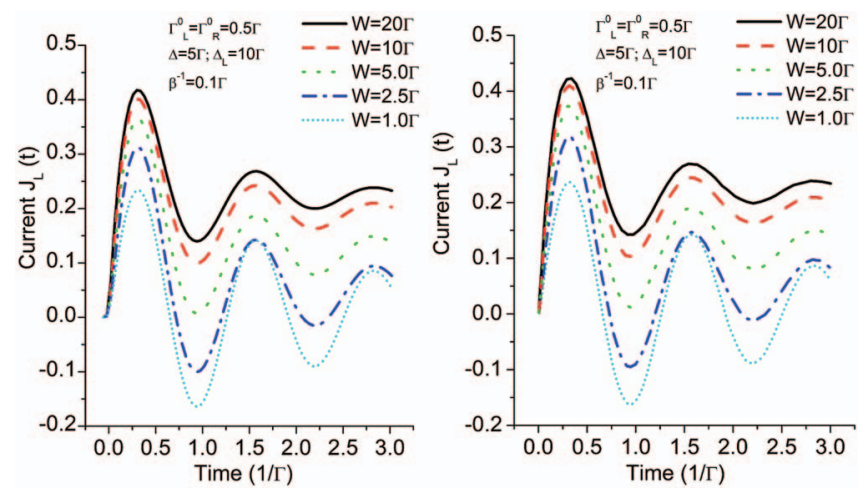

FIG. 2. (Left) Transient currents for single level system with different W values; (Right) Transient currents for the same system reported in Ref. 34.

by the linewidth function as a single Lorentzian

$$
\Lambda_{\alpha}(\varepsilon)=\frac{\Gamma_{\alpha}^{0} W^{2}}{\varepsilon^{2}+W^{2}},
$$

where $\Gamma_{\alpha}^{0}\left(\Gamma_{L}^{0}=\Gamma_{R}^{0}=0.5 \Gamma\right)$ is the linewidth amplitude and $\mathrm{W}$ is the bandwidth. The step-function bias is applied on two leads at time $t=0$. After $t=0$, the left lead has the potential of $10 \Gamma$ and the right lead keeps the potential grounded. The onsite energy is also a step-function, which is $5 \Gamma$ after the time $t$ $=0 . k_{B} T$ is set to $0.1 \Gamma$ for both reservoirs. The Fermi function is expressed with the Padè $(10,10)$ spectrum decomposition. Figure 2 (left) shows our calculated current (left lead) with five different spectral widths: $W=20 \Gamma, 10 \Gamma, 5 \Gamma, 2.5 \Gamma, 1.0 \Gamma$. We compare our work with the exact numerical calculation reported in Ref. 34 (see Fig. 2 right), it is apparent that our calculations recover perfectly the results in Ref. 34 .

\section{B. Simulation of transient current through a chain of atoms}

In our calculation, we fit the self-energy matrix with multiple Lorentzian expansion. For 1D tight-binding model with the nearest neighbor interaction, the surface Green's function and linewidth function can be calculated analytically. There are two approaches. One employs a summation scheme, based on the following: $:^{4,35}$

$$
g\left(l_{1}, l_{2}, z\right)=\sum_{k} \frac{\psi_{k}^{*}\left(l_{1}\right) \cdot \psi_{k}\left(l_{2}\right)}{z-\varepsilon(k)},
$$

where $\psi_{k}(l)=\sin (k a l)$ is the eigenfunction with the wave vector $\mathrm{k}$ for the semi-infinite chain; $\varepsilon(k)$ is the eigenenergy, $z$ is the energy, which can be extended into the complex plane. The details are referred in the literature. ${ }^{4,35}$ Another approach uses Dyson's equation. This can be seen from the book of Cuevas and Scheer. ${ }^{4}$ Since here we treat the nearest neighbor TB model, only $g_{11}^{r}(E)$ is used for the self-energy. The analytical result of $g_{11}^{r}(E)$ is

$$
g_{1,1}^{r}(E)=\frac{1}{t_{0}}\left(\left(\frac{E-\varepsilon_{0}}{2 t_{0}}\right)-i \sqrt{1-\left(\frac{E-\varepsilon_{0}}{2 t_{0}}\right)^{2}}\right),
$$

where $t_{0}$ is the coupling constant (positive) and $\varepsilon_{0}$ is the onsite energy of each site. With $g_{1,1}^{r}(E)$ we can obtain the self-
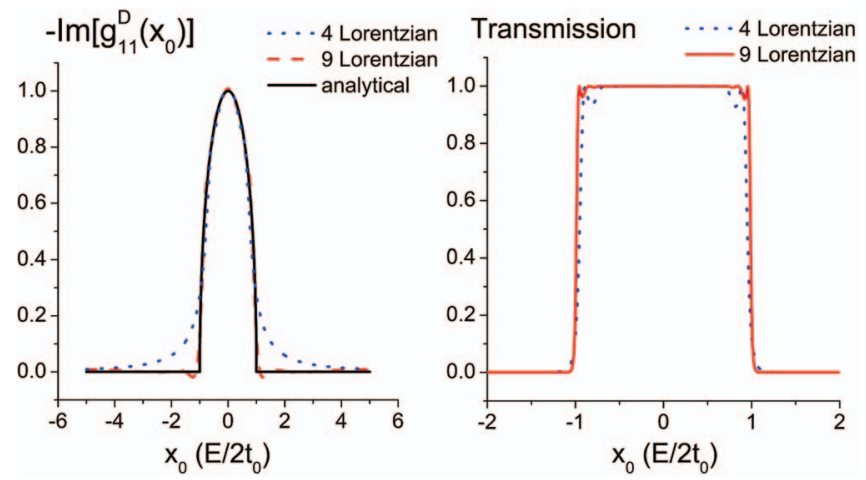

FIG. 3. Left: Lorentzian fitting curves (dotted line for 4 Lorentzian fitting and dashed line for 9 Lorentzian fitting) and the accurate curve (solid line) for the dimensionless linewidth function $\left(-\operatorname{Im}\left[g_{1,1}^{D}\left(x_{0}\right)\right]\right)$; Right: transmission spectrum of 4-site chain TB system calculated with the 4-Lorentzian fitted self-energy (dotted line) and 9-Lorentzian fitted self-energy (solid line).

energy, as well as the linewidth function. To fit $g_{1,1}^{r}(E)$, we factorize out $1 / t_{0}$ in the left side of Eq. (24), to obtain a dimensionless quantity $\left(g_{1,1}^{D}\left(x_{0}\right)\right)$ with the dimensionless variable $x_{0}$ $\left(x_{0}=\frac{E-\varepsilon_{0}}{2 t_{0}}\right)$

$$
g_{1,1}^{D}\left(x_{0}\right)=\operatorname{tg}_{1,1}^{r}(E)=\left(x_{0}-i \sqrt{1-x_{0}^{2}}\right) .
$$

MINPACK for the least-squares method is employed in the multi-Lorentzian fitting calculation. ${ }^{36}$

The left panel of Fig. 3 shows the fitting results (with 4and 9-Lorentzians) and the analytical result of $-\operatorname{Im}\left[g_{1,1}^{D}\left(x_{0}\right)\right]$ for a 1D nearest neighbor TB model (we set the on-site energy $\varepsilon_{0}=0$ ). We observe that the curve for 9-Lorentzians is much closer to the analytical result as compared to that of 4-Lorentzians. The 9-Lorentzian fitting is thus used as the highly accurate scheme. The right panel of Fig. 3 shows the transmission spectrum for 1D TB chain (4-site device), calculated using the following formula: ${ }^{4}$

$$
\begin{gathered}
T=\operatorname{tr}\left[\mathbf{G}^{r} \boldsymbol{\Lambda}_{L} \mathbf{G}^{a} \boldsymbol{\Lambda}_{R}\right], \\
\mathbf{G}^{r}(E)=\left(E \cdot \mathbf{I}-\mathbf{H}-\boldsymbol{\Sigma}^{r}(E)\right)^{-1},
\end{gathered}
$$

where the self-energy $\left(\boldsymbol{\Sigma}^{r}\right)$ and linewidth function $\left(\boldsymbol{\Lambda}_{L}, \boldsymbol{\Lambda}_{R}\right)$ are expanded in terms of multiple Lorentzians. The real part of the self-energy can be obtained from the imaginary part, i.e., the linewidth function, by employing the Kramer-Kronig relation. ${ }^{37}$ The dashed and solid lines are the spectra for 4and 9-Lorentzian fittings, respectively. Since the entire system containing the electrodes and device constitute a homogeneous chain, the transmission coefficient is 1 within $(-2 t, 2 t)$. The transmission spectrum is a rectangular curve. It is clear that the transmission spectrum for the 9-Lorentzian fitting is much closer to the exact values than that of 4-Lorentzians.

We calculate the transient currents using two fitting schemes. Figure 4 shows the comparison results for a 4 -site system. The currents are induced by a bias voltage with a step function at time $\mathrm{t}=0$ and an amplitude of $0.01 \mathrm{~V}$. 4(or 9-) Lorentzians and 10 Padè points are used in fitting the linewidth and Fermi functions, respectively. The coupling constant within the device or the leads is $t_{0}(2 \mathrm{eV})$; and between leads and the device, is $0.9 t_{0}(1.8 \mathrm{eV})$. The temperature 


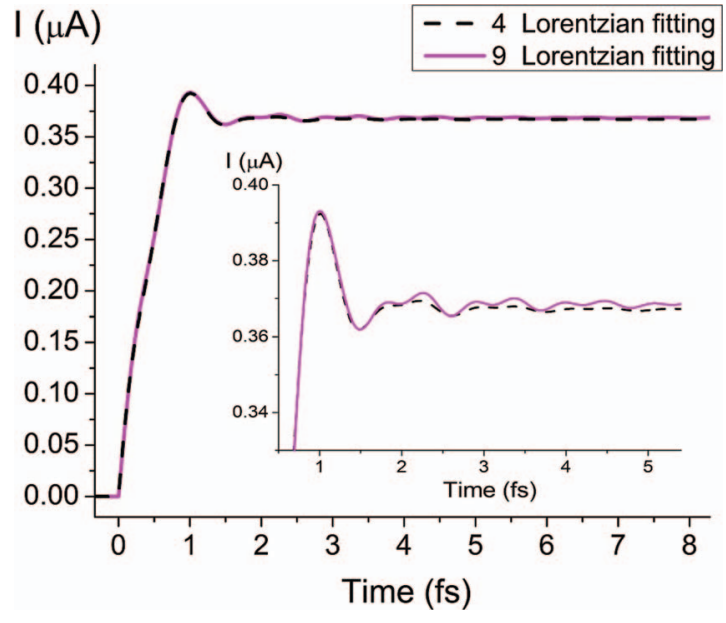

FIG. 4. Time-dependent current for a 4-site 1D system with two linewidth fitting schemes: 4-Lorentzian (dashed line) and 9-Lorentzian (solid line) fitting. The inset is the magnified figure. The coupling constant: in the leads and device, $t_{0}=2 \mathrm{eV}$; between leads and device $h=0.9 t_{0}$. Temperature is 300 $\mathrm{K}$, bias voltage is $0.01 \mathrm{~V}, 10$ Padè points are used.

is $300 \mathrm{~K}$. The bias is symmetrically applied on two leads, and the site energy in the device varies linearly between the two leads.

We note that the current curves by two fitting schemes are almost the same. The tiny difference is observed from the magnified figure (the inset of Fig. 4) that the transient current by 9 -Lorentzian has more high frequency oscillations than the current by 4-Lorentzian fitting. It seems strange that although the linewidth functions from these two fittings are almost the same in such a small bias range $(0.01 \mathrm{~V})$, there still exist different transient currents. This would be explained in the following. The sharply changed step bias in time domain involves a wide frequency range of Fourier components. On the other hand, the lead can transmit the waves of the frequencies within their linewidth energy range. Since the 4-Lorentzian fitted linewidth function is wider than the 9-Lorentzian one (on the tails, far from the center), they can dissipate higher frequency components of bias signal. So the current from the 4-Lorentzian linewidth has less high frequency oscillations and stronger damping. This phenomenon was also mentioned by other authors, ${ }^{38}$ and it shows that the transient current depends on the linewidth-function behavior on the whole energy range.

\section{Simulation of one-dimensional atom chain with different contacts}

We investigate the 1D atom chain with good and poor device/lead coupling. For the device with 2 atoms, the site energy linearly changes between two leads and a symmetric bias voltage of $2 \mathrm{~V}$ is applied on two leads. With the nearest neighbor TB model, the coupling constants $t$ in leads and device are $2 \mathrm{eV}$; and $t$ from leads to device is $1.8 \mathrm{eV}$ (good contact) or $0.4 \mathrm{eV}$ (poor contact). For the linewidth function and Fermi function, 4-Lorentzian fitting and 10 Padè poles are used, respectively. The temperature is $300 \mathrm{~K}$.

Figure 5 shows the I-V curve (left), density of states (DOS) spectrum (middle), and the transient current behav-
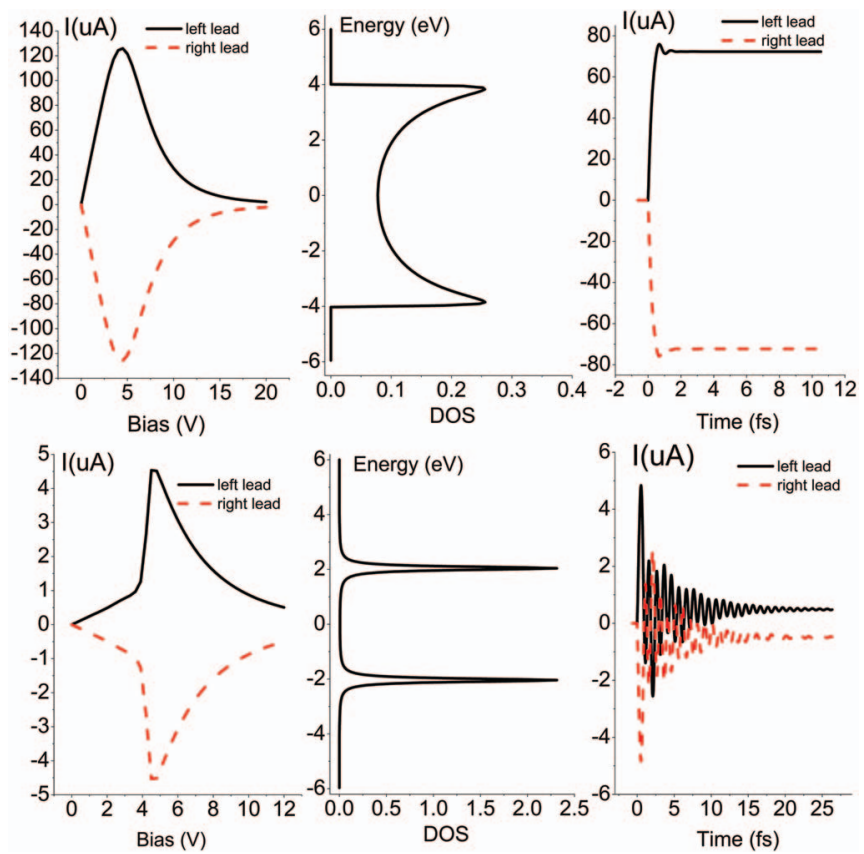

FIG. 5. I-V curve (left), DOS spectrum (middle), and the transient current (right) for a 2-atom device with good contact (upper row) and poor contact (lower row). The bias voltage is $2 \mathrm{~V}$, the coupling constants $t$ in leads and device are $2 \mathrm{eV}$; and $t$ from leads to device is $1.8 \mathrm{eV}$ (good contact) or $0.4 \mathrm{eV}$ (poor contact).

ior (right) for the good contact (upper row) and poor contact (lower row) cases, respectively.

First, we focus on the good contact case. From the I-V curve, we know that at a small bias the curve is linear but at a larger bias (greater than $5 \mathrm{~V}$ ) the curve bends down, which means a negative differential conductance (NDC). ${ }^{39}$ This type of NDC, not the common ones resulting from the Bloch scattering in super-lattice structures, is caused by the finite spectrum width of leads. As the total current is determined by the overlap of the occupied and unoccupied states in two leads, under a larger bias this overlap decreases and the lead current decreases. Figure 5 (middle) shows the DOS of the device under zero bias. It is very similar to the DOS of an infinite chain, ${ }^{35}$ since this model is a good contact chain. And from the time-dependent current curve, we see that after about $2 \mathrm{fs}$, the current reaches the steady state.

Second, we investigate the poor contact case. We see the $\mathrm{I}-\mathrm{V}$ curve increased with a much larger slope at a bias about 4 $\mathrm{V}$; and the DOS spectrum has very sharp peaks. These are due to the small self-energy for poor contact. Since self-energy can widen the DOS of device (DOS is series of delta functions without the self-energy), the small self-energy makes the sharp peaks of DOS. Only when the center of DOS levels approach to the lead energy levels, can the current rise. Thus, this sharp-peak DOS explains the abrupt rise in the I-V curve. For the transient current, there is large and long oscillation before achieving a steady state. This is also due to the weak coupling. In the weak coupling, it is difficult for electrons to transmit between the leads and device. So they oscillate in device with a small damping before reaching a steady state. 


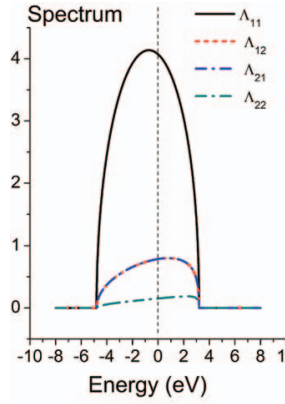

(a)

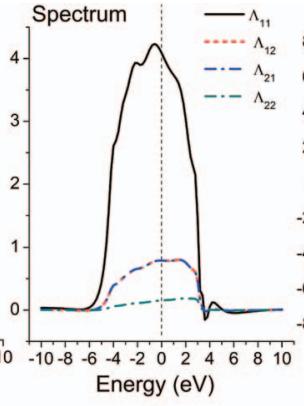

(b)

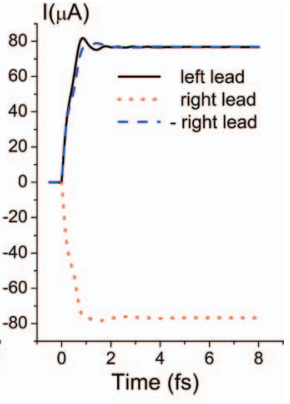

(c)
FIG. 6. The linewidth functions with the lead Green's functions obtained from the accurate iteration method (a) or from the Lorentzian fitting (b) scheme. ( $\Lambda_{12}$ and $\Lambda_{21}$ correspond the same curves.) The system is a 4site TB model with the next nearest neighbor interactions. The parameters: $\mathrm{t}=2 \mathrm{eV} ; \mathrm{h}_{2}=0.4 \mathrm{eV}$. (c) The transient current of the system under a step bias voltage $(2 \mathrm{~V})$ symmetrically applied on two leads at time $=0$. The dotted line is the mirror (opposite sign) of the dashed line (right current).

\section{Tight-binding model with the next nearest neighbor interactions and asymmetric left-and-right transient current}

We also use our HEOM to the TB model with the next nearest neighbor interactions. The system is a 4-site atom chain with the next nearest neighbor interactions in all the regions. The nonzero elements of the lead/device coupling matrix are: $h_{i, i \pm 1}=\mathrm{t}=2 \mathrm{eV} ; h_{i, i \pm 2}=\mathrm{h}_{2}=0.4 \mathrm{eV}$. Figure 6(a) shows the linewidth functions with accurate results, calculated from Eq. (18). The surface Green's functions of the lead are calculated with an iteration method, as mentioned in Refs. 40 and 41. Figure 6(b) shows the Lorentzian fitted linewidth functions, calculated from Eq. (21). Totally 22 Lorentzian functions are used: 9 Lorentzians for $\mathrm{g}_{11}$ and $\mathrm{g}_{12}, 4$ Lorentzians for $\mathrm{g}_{22}$.

Compared to Fig. 3 (left), we see that the linewidth functions like $\Lambda_{11}$ are asymmetric about $\mathrm{E}=0$. This is because that in the next nearest neighbor model, $\Lambda_{11}$ involves three types of the surface Green's functions: $g_{11}, g_{12}$, and $g_{22}$. In the lead region with the next nearest neighbor interactions, these three Green's functions are asymmetric, which makes such asymmetric linewidth functions.

From Fig. 6(c), we find that when a bias voltage (2 V) is symmetrically applied on two leads at time $=0$, their transient currents are not symmetric! This seems strange since the system is symmetric about two leads. The reason lies in the asymmetric linewidth functions: We know in the linewidth function curve, the area above $\mathrm{E}=0$ represents the amount of electrons that one lead can afford to the device; and the area below $\mathrm{E}=0$ represents the amount of unoccupied orbitals for the other lead to accept electrons. So this asymmetric property in energy dimension results in the asymmetric transient currents in two leads.

\section{NUMERICAL EFFICIENCY}

Most numerical methods in quantum dissipation theory including the RMDM-HEOM method are computationally expensive, and are thus limited to model systems. As the RSDM is a physical quantity with much reduced dimension,
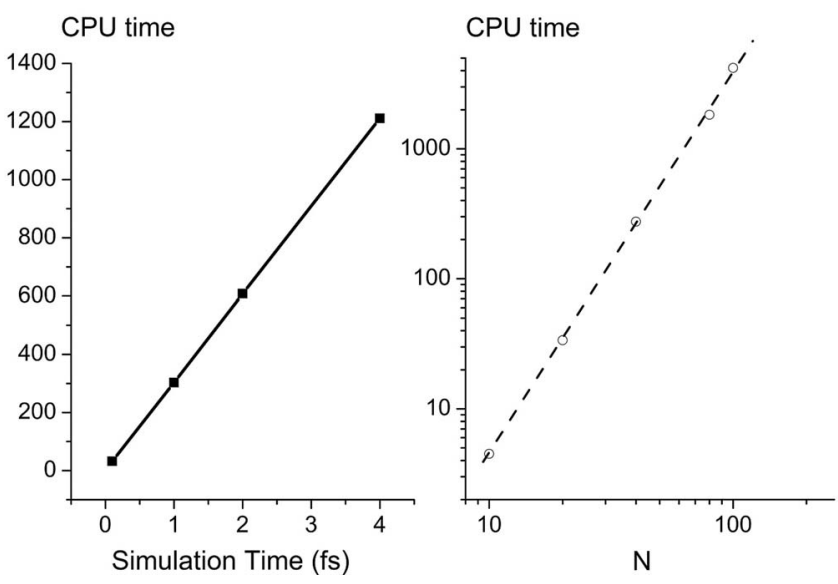

FIG. 7. CPU time dependence on the simulation time (left) and site number $\mathrm{N}$ (right). In the left figure, the system is a 1D TB chain with 20 sites, 4 Lorentzians and 10 Padè points. And in the right figure, the simulation time is fixed at $0.1 \mathrm{fs}$, and the time step is $0.002 \mathrm{fs}$ for these two cases.

the numerical solution of our RSDM-HEOM method is expected to be much more efficient.

The bottleneck of calculation is the large number of elements for second-tier ADM, which is proportional to $N^{2}\left(N_{\alpha} N_{k}\right)^{2}$, where $N$ is the number of orbitals in the system of interest, $N_{\alpha}$ is the number of leads, and $N_{k}$ is the total number of expansion. So in order to reduce the computation costs, we need to use small number of Lorentzian/Padè terms.

In the RSDM-HEOM approach, three hierarchical differential equations are integrated in time domain with the fourthorder Runge-Kutta technique. The total computation time is proportional to the simulation time, as is shown in Fig. 7 (left). This is more efficient than the conventional NEGF methods, since even for the Green function the convolution on time domain is needed, which reflects the memory. The computational time scales as $\mathrm{O}\left(N^{3}\right)$ with $N$, the number of the orbitals, and this is shown in Fig. 7 (right). This is because that the most consuming computation is the matrix-matrix multiplication. Each matrix, such as $\boldsymbol{\sigma}(t)$ and $\mathbf{A}_{\alpha k}^{>+}$, has the dimension of $N$. Thus, the computational time, or the central processing unit (CPU) time, is proportional to $N^{3}$.

\section{CONCLUSION}

We have implemented a practical numerical method for the single-electron Liouville-von-Neumann equation. It goes beyond the WBL approximation. The self-energy decomposition technique is used to transform the integrations in the RSDM-HEOM to the residue summations. The Lorentzian-Padè decomposition scheme is employed to evaluate the linewidth matrix and Fermi function, respectively. The RSDM-HEOM method developed here is applicable to any effective one-electron models or Hamiltonians such as DFT and density functional tight-binding (DFTB). To illustrate the validity of our method, we simulate the two- and four-atom systems with different Lorentzian fitting schemes, and in the nearest and next nearest neighbor tight-binding models. To demonstrate its efficiency, we have carried out the simulations on the systems containing up to one hundred of atoms, and 
confirmed its computation time scales linearly with the physical simulation time and cubically with the system size.

An important motivation of our work is to develop a practical first-principles Liouville-von Neumann equation for open systems, and thus combine the first-principles method and quantum dissipation theory. The method developed here can be readily implemented at first-principles level, i.e., TDDFT, as TDDFT can be formulated in terms of the RSDM as well. ${ }^{42}$ Our work constitutes thus a major step toward such a goal.

\section{ACKNOWLEDGMENTS}

Authors would like to thank Yu Zhang and Hui Cao for many helpful discussions on transport theory and spectral integral. The authors also give thanks to Dr. Jian Sun for some help with computer. Support from the Hong Kong Research Grant Council (HKU700808P, HKU700909P, HKU700711P, HKUST9/CRF/08), AOE (AOE/P-04/08), National Science Foundation of China (NSFC) (21103157, 21033008), and Fundamental Research Funds for the Central Universities of China (2340000034) is gratefully acknowledged.

\section{APPENDIX: HEOM DERIVATION FROM NEGF SCHEME}

1. Energy resolution scheme

From NEGF theory, we can define the auxiliary density matrix,

$$
\begin{gathered}
\boldsymbol{\varphi}_{\alpha}(t)=i \int_{-\infty}^{t} d \tau\left[\mathbf{G}_{D}^{<}(t, \tau) \cdot \boldsymbol{\Sigma}_{\alpha}^{>}(\tau, t)\right. \\
\left.-\mathbf{G}_{D}^{>}(t, \tau) \cdot \boldsymbol{\Sigma}_{\alpha}^{<}(\tau, t)\right],
\end{gathered}
$$

so the EOM of density matrix [Eq. (2)] is rewritten as

$$
i \dot{\sigma}_{D}(t)=\left[\mathbf{h}_{D}(t), \sigma_{D}(t)\right]-\sum_{\alpha}\left[\boldsymbol{\varphi}_{\alpha}(t)-\boldsymbol{\varphi}_{\alpha}^{\dagger}(t)\right] .
$$

If we introduce the energy-resolved self-energy, $\boldsymbol{\Sigma}_{\alpha}^{<,>}(\varepsilon, \tau, t), \boldsymbol{\Sigma}_{\alpha}^{<,>}(\tau, t)=\int d \varepsilon \cdot \boldsymbol{\Sigma}_{\alpha}^{<,>}(\varepsilon, \tau, t)$ and the energy-resolved auxiliary density matrix of the first-tier, $\boldsymbol{\varphi}_{\alpha}(\varepsilon, t), \boldsymbol{\varphi}_{\alpha}(t)=\int d \varepsilon \cdot \boldsymbol{\varphi}_{\alpha}(\varepsilon, t)$, Eq. (A2) can be rewritten as the differential-integral form as shown in Eq. (5). $\boldsymbol{\varphi}_{\alpha}(\varepsilon, t)$ definition is given in Eq. (3).

2. EOMs of $\boldsymbol{\Sigma}_{\alpha}^{<,>}(\varepsilon, \tau, t)$ and $\mathbf{G}_{D}^{<,>}(t, \tau)$

As discussed in the Jauho and Wingreen's paper, ${ }^{7}$ the electron in the lead is viewed as non-interacting except for a self-consistent potential. The occupation of each state is determined by an equilibrium distribution function. So the time-dependent lesser Green's function for state $k \alpha$ is written as

$$
g_{k \alpha}^{<}\left(t, t^{\prime}\right)=i f\left(\varepsilon_{k \alpha}^{0}\right) \exp \left[-i \int_{t^{\prime}}^{t} d t_{1} \varepsilon_{k \alpha}\left(t_{1}\right)\right],
$$

where $\varepsilon_{k \alpha}\left(t_{1}\right)=\varepsilon_{k \alpha}^{0}+\Delta_{\alpha}(t), \varepsilon_{k \alpha}^{0}$ is the equilibrium energy for $\mathrm{k}$ state and $\Delta_{\alpha}(t)$ is the time-dependent bias potential in lead $\alpha$.
Since the self-energy is related to the lead Green's function by the device-lead coupling matrix, from Eq. (A3), the lesser/greater self-energy can be written as

$$
\boldsymbol{\Sigma}_{\alpha}^{<,>}(t, \tau)=e^{-i \int_{\tau}^{t} \Delta_{\alpha}(\xi) d \xi} \cdot \widetilde{\boldsymbol{\Sigma}}_{\alpha}^{<,>}(t-\tau),
$$

where $\widetilde{\boldsymbol{\Sigma}}_{\alpha}^{<,>}(t, \tau)$ refers to the steady-state self-energy, in which a single-time variable is used $\left(\widetilde{\boldsymbol{\Sigma}}_{\alpha}^{<,>}(t, \tau)\right.$ $\left.=\widetilde{\boldsymbol{\Sigma}}_{\alpha}^{<,>}(t-\tau)\right)$, and the phase factor $e^{-i \int_{\tau}^{t} \Delta_{\alpha}(\xi) d \xi}$ reflects the influence of the bias potential. $\widetilde{\boldsymbol{\Sigma}}_{\alpha}^{<,>}(t, \tau)$ may be expressed in the Fourier integral form

$$
\widetilde{\boldsymbol{\Sigma}}_{\alpha}^{<}(\tau-t)=\frac{i}{2 \pi} \int_{-\infty}^{+\infty} f_{\alpha}(\varepsilon) \boldsymbol{\Lambda}_{\alpha}(\varepsilon) \cdot e^{i \varepsilon(t-\tau)} d \varepsilon,
$$

$$
\widetilde{\mathbf{\Sigma}}_{\alpha}^{>}(\tau-t)=\frac{-i}{2 \pi} \int_{-\infty}^{+\infty}\left(1-f_{\alpha}(\varepsilon)\right) \boldsymbol{\Lambda}_{\alpha}(\varepsilon) \cdot e^{i \varepsilon(t-\tau)} d \varepsilon,
$$

where $\boldsymbol{\Lambda}_{\alpha}(\varepsilon)$ is the linewidth function, and $f_{\alpha}(\varepsilon)$ is the Fermi function for lead $\alpha$.

Thus, the energy-resolved self-energy is

$$
\boldsymbol{\Sigma}_{\alpha}^{<,>}(\varepsilon, t, \tau)=e^{-i \int_{\tau}^{t} \Delta_{\alpha}(\xi) d \xi} \widetilde{\boldsymbol{\Sigma}}_{\alpha}^{<,>}(\varepsilon, t-\tau),
$$

where $\widetilde{\boldsymbol{\Sigma}}_{\alpha}^{<,>}(\varepsilon, t-\tau)$ is the integrand in Eqs. (A5). From this definition, it is easy to derive the EOMs of $\boldsymbol{\Sigma}_{\alpha}^{<,>}(\varepsilon, t, \tau)$

$$
\begin{gathered}
\frac{\partial \boldsymbol{\Sigma}_{\alpha}^{<,>}(\varepsilon, t, \tau)}{\partial t}=-i\left[\varepsilon+\Delta_{\alpha}(t)\right] \boldsymbol{\Sigma}_{\alpha}^{<,>}(\varepsilon, t, \tau), \\
\frac{\partial \boldsymbol{\Sigma}_{\alpha}^{<,>}(\varepsilon, t, \tau)}{\partial \tau}=i\left[\varepsilon+\Delta_{\alpha}(t)\right] \boldsymbol{\Sigma}_{\alpha}^{<,>}(\varepsilon, t, \tau) .
\end{gathered}
$$

The EOM of Green's functions $\mathbf{G}_{D}^{<,>}(t, \tau)$ can be derived from the Dyson equation,

$$
\begin{aligned}
i \frac{\partial \mathbf{G}_{D}^{<,>}(t, \tau)}{\partial t} & \\
= & \mathbf{h}_{D} \mathbf{G}_{D}^{<,>}(t, \tau)+\int_{-\infty}^{+\infty} d t_{1}\left[\boldsymbol{\Sigma}_{\text {total }}^{<,>}\left(t, t_{1}\right) \cdot \mathbf{G}_{D}^{a}\left(t_{1}, \tau\right)\right. \\
& \left.+\boldsymbol{\Sigma}_{\text {total }}^{r}\left(t, t_{1}\right) \cdot \mathbf{G}_{D}^{<,>}\left(t_{1}, \tau\right)\right],
\end{aligned}
$$

where $\boldsymbol{\Sigma}_{\text {total }}^{<,>, r}$ is the total self-energy for all leads connecting to the device, since $\mathbf{G}_{D}^{<,>}(t, \tau)$ is related to the device. The following relation is used in the derivation:

$$
\begin{aligned}
\boldsymbol{\Sigma}_{\text {total }}^{<,>r}(t, \tau) & =\sum_{\alpha^{\prime}}^{N_{\alpha}} \boldsymbol{\Sigma}_{\alpha^{\prime}}^{<,>, r}(t, \tau) \\
& =\sum_{\alpha^{\prime}}^{N_{\alpha}} \int d \varepsilon^{\prime} \cdot \boldsymbol{\Sigma}_{\alpha^{\prime}}^{<,>, r}\left(\varepsilon^{\prime}, t, \tau\right) .
\end{aligned}
$$

3. EOM of auxiliary density matrices

Utilizing these EOMs for $\boldsymbol{\Sigma}_{\alpha}^{<,>}(\varepsilon, t, \tau)$ and $\mathbf{G}_{D}^{<,>}(t, \tau)$, we may derive the $\mathrm{EOM}$ of $\varphi_{\alpha}(\varepsilon, t)$. In the derivation, the derivative operator $\partial_{t}$ acts in three 
positions of $\boldsymbol{\varphi}_{\alpha}(\varepsilon, t)$ expression [Eq. (3)]: (a) on the integration symbol, and cancel this operation; (b) on $\boldsymbol{\Sigma}_{\alpha}^{>,<}(\varepsilon, \tau, t), \quad \boldsymbol{\Sigma}_{\alpha}^{>,<}(\varepsilon, \tau, t)$ is replaced by $\partial_{t} \boldsymbol{\Sigma}_{\alpha}^{>,<}(\varepsilon, \tau, t)$; and (c) on $\mathbf{G}_{D}^{<,>}(t, \tau), \mathbf{G}_{D}^{<,>}(t, \tau)$ is replaced by $\partial_{t} \mathbf{G}_{D}^{<,>}(t, \tau)$. In part (c), the expansion form of $\Sigma_{\text {total }}^{<,>, r}$ [Eq. (A9)] is used and the terms like $\int_{-\infty}^{t}$ $d t_{1} \int_{-\infty}^{t} d t_{2} \boldsymbol{\Sigma}_{\alpha^{\prime}}^{<}\left(\varepsilon^{\prime}, t, t_{1}\right) \cdot \mathbf{G}_{D}^{a}\left(t_{1}, t_{2}\right) \boldsymbol{\Sigma}_{\alpha}^{>}\left(\varepsilon, t_{2}, t\right) \quad$ appears, which results in ADM of the second-tier, $\boldsymbol{\varphi}_{\alpha \alpha^{\prime}}(\varepsilon$, $\varepsilon^{\prime}, t$ ), as defined in Eq. (4).

After some rearrangement and simplification, the EOM of $\boldsymbol{\varphi}_{\alpha}(\varepsilon, t)$ is obtained, as shown in Eq. (6). And with the similar approach, the EOM of $\varphi_{\alpha \alpha^{\prime}}\left(\varepsilon, \varepsilon^{\prime}, t\right)$ can be derived, as shown in Eq. (7).

\section{Discrete version of HEOM}

From Eqs. (9) and (11), we know that $\widetilde{\boldsymbol{\Sigma}}_{\alpha}^{<,>}(\tau-t)$ is transformed into the summation form. With a phase factor, $\boldsymbol{\Sigma}_{\alpha}^{<,>}(\tau, t)$ can also be expressed as the summation form, $\Sigma_{\alpha}^{<,>}(\tau, t)=\sum_{k}^{N_{k}} \boldsymbol{\Sigma}_{\alpha k}^{<,>}(\tau, t)$. Each discrete component is

$$
\boldsymbol{\Sigma}_{\alpha k}^{<,>}(\tau, t)=\mathbf{A}_{\alpha k}^{<,> \pm} e^{\mp \gamma_{\alpha k}^{ \pm}(t-\tau)} \cdot \mathrm{e}^{i \int_{\tau}^{t} \Delta_{\alpha}(\xi) \xi} .
$$

From this, it is easy to obtain the EOMs for $\Sigma_{\alpha k}^{<,>}\left(t_{1}, t_{2}\right)$ ("+" corresponds to $t_{1}-t_{2}<0$ and "-" corresponds to $\left.t_{1}-t_{2}>0\right)$,

$$
\begin{aligned}
& \frac{\partial \boldsymbol{\Sigma}_{\alpha k}^{<,> \pm}\left(t_{1}, t_{2}\right)}{\partial t_{1}}=\left[ \pm \gamma_{\alpha k}^{ \pm}-i \Delta_{\alpha}(t)\right] \boldsymbol{\Sigma}_{\alpha k}^{<,> \pm}\left(t_{1}, t_{2}\right), \\
& \frac{\partial \boldsymbol{\Sigma}_{\alpha k}^{<,> \pm}\left(t_{1}, t_{2}\right)}{\partial t_{2}}=\left[\mp \gamma_{\alpha k}^{ \pm}+i \Delta_{\alpha}(t)\right] \boldsymbol{\Sigma}_{\alpha k}^{<,> \pm}\left(t_{1}, t_{2}\right) .
\end{aligned}
$$

(A11b)

These two groups ("+" and "-") of EOMs are used in two difference cases, as discussed in Sec. II B.

Similar to the self-energies, $\varphi_{\alpha}(t)$ can be written as the summation instead of integration as well,

$$
\boldsymbol{\varphi}_{\alpha}(t)=\int d \varepsilon \cdot \boldsymbol{\varphi}_{\alpha}(\varepsilon, t)=\sum_{k=1}^{N_{k}} \boldsymbol{\varphi}_{\alpha k}(t)
$$

and the second-tier $\operatorname{ADM} \boldsymbol{\varphi}_{\alpha \alpha^{\prime}}\left(\varepsilon, \varepsilon^{\prime}, t\right)$ can be expressed as the sum of the discrete second-tier terms as

$$
\boldsymbol{\varphi}_{\alpha \alpha^{\prime}}(t)=\iint d \varepsilon d \varepsilon^{\prime} \cdot \boldsymbol{\varphi}_{\alpha \alpha^{\prime}}\left(\varepsilon, \varepsilon^{\prime}, t\right)=\sum_{k, k^{\prime}}^{N_{k}} \boldsymbol{\varphi}_{\alpha k, \alpha^{\prime} k^{\prime}}(t) .
$$

The definitions of these discrete ADMs are similar to $\boldsymbol{\varphi}_{\alpha}(\varepsilon, t)$ and $\boldsymbol{\varphi}_{\alpha \alpha^{\prime}}\left(\varepsilon, \varepsilon^{\prime}, t\right)$ definitions (Eqs. (3) and (4)), with $\boldsymbol{\Sigma}_{\alpha}^{<,>}\left(\varepsilon, t_{1}, t_{2}\right)$ replaced by $\boldsymbol{\Sigma}_{\alpha k}^{<,>}\left(t_{1}, t_{2}\right)$. By the same scheme as mentioned before, the EOMs of $\boldsymbol{\varphi}_{\alpha k}(t)$ and $\boldsymbol{\varphi}_{\alpha k, \alpha^{\prime} k^{\prime}}(t)$ can be obtained, as shown in Eqs. (15)-(17).

${ }^{1}$ M. Auf der Maur, M. Povolotskyi, F. Sacconi, A. Pecchia, G. Romano, G. Penazzi, and A. Di Carlo, Opt. Quantum Electron. 40, 1077 (2008).

${ }^{2}$ T. Fujisawa, D. G. Austing, Y. Tokura, Y. Hirayama, and S. Tarucha, J. Phys.: Condens. Matter 15, R1395 (2003).

${ }^{3}$ T. Hayashi, T. Fujisawa, H. D. Cheong, Y. H. Jeong, and Y. Hirayama, Phys. Rev. Lett. 91, 226804 (2003).
${ }^{4}$ J. C. Cuevas and E. Scheer, Molecular Electronics: An Introduction to Theory and Experiment (World Scientific, 2001); see also S. Datta, Electronic Transport in Mesoscopic Systems (Cambridge University Press, 1995).

${ }^{5}$ Y. Xue, S. Datta, and M. A. Ratner, Chem. Phys. 281, 151 (2002).

${ }^{6}$ J. Taylor, H. Guo, and J. Wang, Phys. Rev. B 63, 245407 (2001).

${ }^{7}$ A. P. Jauho, N. S. Wingreen, and Y. Meir, Phys. Rev. B 50, 5528 (1994).

${ }^{8}$ Y. Zhu, J. Maciejko, T. Ji, H. Guo, and J. Wang, Phys. Rev. B 71, 075317 (2005).

${ }^{9}$ E. Runge and E. K. U. Gross, Phys. Rev. Lett. 52, 997-1000 (1984).

${ }^{10}$ K. Burke, R. Car, and R. Gebauer, Phys. Rev. Lett. 94, 146803 (2005).

${ }^{11}$ R. Gebauer, K. Burke, and R. Car, Lect. Notes Phys. 706, 463 (2006).

${ }^{12}$ X. Zheng, F. Wang, C. Y. Yam, Y. Mo, and G. H. Chen, Phys. Rev. B. 75, 195127 (2007).

${ }^{13}$ C. Y. Yam, Y. Mo, F. Wang, X. B. Li, G. H. Chen, X. Zheng, Y. Matsuda, J. Tahir-Kheli, W. A. Goddard III, Nanotechnology 19, 495203 (2008)

${ }^{14}$ J. Yuen-Zhou, D. G. Tempel, C. A. Rodriguez-Rosario, and A. AspuruGuzik, Phys. Rev. Lett. 104, 043001 (2010).

${ }^{15}$ C. Y. Yam, X. Zheng, G. H. Chen, Y. Wang, T. Frauenheim, and T. A. Niehaus, Phys. Rev. B 83, 245448 (2011).

${ }^{16}$ S. H. Ke, R. Liu, W. Yang, and H. U. Baranger, J. Chem. Phys. 132, 234105 (2010).

${ }^{17}$ S. Z. Wen, S. K. Koo, C. Y. Yam, X. Zheng, Y. J. Yan, Z. M. Su, K. N. Fan, L. Cao, W. P. Wang, and G. H Chen, J. Phys. Chem. B 115, 5519 (2011).

${ }^{18}$ G. Stefanucci and C.-O. Almbladh, Europhys. Lett. 67, 14 (2004).

${ }^{19}$ G. Stefanucci and C.-O. Almbladh, Phys. Rev. B 69, 195318 (2004).

${ }^{20}$ G. Stefanucci, C.-O. Almbladh, S. Kurth, E. K. U. Gross, A. Rubio, R. van Leeuwen, N. E. Dahlen, and U. von Barth, Lect. Notes Phys. 706, 479 (2006).

${ }^{21}$ M. Galperin and S. Tretiak, J. Chem. Phys. 128, 124705 (2008).

${ }^{22}$ M. Koentopp, C. Chang, K. Burke, and R. Car, J. Phys.: Condens. Matter 20, 083203 (2008).

${ }^{23}$ R. Gaudoin and K. Burke, Phys. Rev. Lett. 93, 173001 (2004).

${ }^{24}$ R. Baer, J. Chem. Phys. 128, 044103 (2008).

${ }^{25}$ S. Kurth, G. Stefanucci, C. O. Almbladh, A. Rubio, and E. K. U. Gross, Phys. Rev. B 72, 035308 (2005).

${ }^{26}$ C. A. Moyer, Am. J. Phys. 72, 351 (2004).

${ }^{27}$ A. Arnold, VLSI Des. 6, 313 (1998)

${ }^{28}$ Y. Tanimura and R. Kubo, J. Phys. Soc. Jpn. 58, 101 (1989)

${ }^{29}$ J. S. Jin, X. Zheng, and Y. J. Yan, J. Chem. Phys. 128, 234703 (2008).

${ }^{30}$ X. Zheng, G. H. Chen, Y. Mo, S. K. Koo, H. Tian, C. Y. Yam, and Y. J. Yan, J. Chem. Phys. 133, 114101 (2010).

${ }^{31}$ J. Hu, M. Luo, F. Jiang, R. X. Xu, and Y. J. Yan, J. Chem. Phys. 134, 244106 (2011).

${ }^{32} \mathrm{~J}$. Mathews and R. L. Walker, Mathematical Methods of Physics (Benjamin, 1970).

${ }^{33}$ L. Zhang, B. Wang, and J. Wang, Phys. Rev. B 84, 115412 (2011).

${ }^{34}$ J. Maciejko, J. Wang, and H. Guo, Phys. Rev. B 74, 085324 (2006).

${ }^{35}$ E. N. Economou, Green's Function in Quantum Physics (Springer, 1983), Chaps 1 and 5.

${ }^{36}$ MINPACK is a subprogram package for the numerical solution of nonlinear equations and nonlinear least square problems. It is written by $\mathrm{J}$. More, B. Garbow, and K. Hillstrom. It is free to download from the website http://www.netlib.org/minpack/. About least-squares, see Ake Bjorck, Numerical Methods for Least Squares Problems (SIAM, Philadelphia, 1996).

${ }^{37}$ J. D. Jackson, Classical Electrodynamics (Wiley, 1999), pp. 332-333. And note that the Lorentzian function as in Eq. (25) actually is the imaginary part of $\frac{\Gamma_{d}}{\left(\varepsilon-\Omega_{d}\right)+i W_{d}}\left(\eta_{d}=-\Gamma_{d} / W_{d}\right)$. So the real part is easily obtained, which satisfies the KK relation with the imaginary part.

${ }^{38}$ A. Croy and U. Saalmann, Phys. Rev. B 80, 245311 (2009).

${ }^{39}$ J. R. Söderström, D. H. Chow, and T. C. McGill, Appl. Phys. Lett. 55, 1094 (1989).

${ }^{40}$ M. P. L. Sancho, J. M. L. Sacho, and J. Rubio, J. Phys. F: Met. Phys. 15, 851 (1985)

${ }^{41}$ J. Henk and W. Schattke, Comput. Phys. Commun. 77, 69 (1993).

${ }^{42}$ C. Y. Yam, S. Yokojima, and G. H. Chen, Phys. Rev. B 68, 153105 (2003); J. Chem. Phys. 119, 8794 (2003); W. Z. Liang, S. Yokojima, D. H. Zhou, and G. H. Chen, J. Phys. Chem. A 104, 2445 (2000); F. Wang, C. Y. Yam, G. H. Chen and K. N. Fan, J. Chem. Phys. 126, 134104 (2007). 